\section{P-207 REGENERATE OUR OWN TRUE SELVES (ROOTS) MODEL OF GROUP THERAPY}

Lorna Richardson, Colette Parfitt, Emma Kirk, Rachael Stevenson, Tracey Taylor. Marie Curie Hospice Liverpool, UK

\subsection{6/bmjspcare-2017-hospice.232}

Background Not all patients fit into the traditional day therapy model. There is a lack of evidence to guide best practice but research highlights the value of self-management and promoting service-user independence. By introducing a flexible and innovative day therapy model we hope to engage a different cohort of patients and meet the needs of an emerging patient profile. A launch of recent key documents has led us to review the services provided.

Aims To launch ROOTS group therapy in September 2017 offering a wider range of groups not available in the community for palliative patients. The new model will offer a more accessible, patient centred and holistic approach with the aim of ultimately improving quality of life, symptom management and independence for a wider range of patients.

Method Local services were reviewed and a literature review was conducted looking at the evidence for group versus individual intervention. Patient interviews and forums informed the steering group of service-users' preferences for day therapy services. Evaluation of referral rates and patient attendance will be undertaken. Furthermore an evaluation of the model through direct service user feedback and validated outcome measures for specific groups will be used.

Results The evaluation of referrals and attendance will seek to demonstrate whether the model delivers an accessible day therapy service, accessed by a diverse patient population. Patients will be invited to feedback on whether ROOTS delivers a more patient- centred approach.

Conclusion This project will provide information to develop a framework in a positive direction and further investigate innovative day therapy models that are more suitable for our emerging patient population. This information will be shared with stakeholders to inform and inspire best practice.

\section{P-208 COMBINED ONCOLOGY AND PALLIATIVE CARE CLINICS; WORKING IN PARALLEL (PART 1)}

1,2Jane Gibbins, ${ }^{2}$ Beccy Benham, ${ }^{2}$ Anna Broadbent, ${ }^{2}$ Amman Mader, ${ }^{1,2}$ Carolyn Campbell, ${ }^{3}$ Rachel Newman, ${ }^{1,2}$ Deborah Stevens, ${ }^{3}$ Joanne Palmer. 'Cornwall Hospice Care, St Austell, UK; ${ }^{2}$ Exeter Medical School, Exeter, UK; ${ }^{3}$ Royal Cornwall Hospital Trust, Truro, UK

\subsection{6/bmjspcare-2017-hospice.233}

Background Early Specialist Palliative Care (SPC) introduced to patients with advanced lung cancer has recently been proven to enhance quality-of-life and improve survival. Combined Oncology and Palliative Care Clinics (COPCC) have been carried at Royal Cornwall Hospital Trust for over two decades, to enable patients to be reviewed by palliative care consultants (PCC) whilst attending the Oncology Centre; enabling the two specialities to run in parallel to improve patient care. The aims of this project were to explore the types of patients seen and content of consultations.

Methods A retrospective review of the current PCC consultation letter of 150 consecutive patients reviewed between 01/ 2016-06/2016. Data collected by three medical students.

Results Of the 150 patients, 49\% had on-going/planned palliative oncological options (POO), 20\% optimal supportive/ symptom control (SSC), $18 \%$ radical treatment and $13 \%$ treated with curative intent. $75 \%$ had stable disease, $23 \%$ deteriorating and $2 \%$ in their last month of life. The main focus of the consultation was symptom control (95\%), followed by discussions around aims of palliative/oncological treatment $(71 \%)$. For patients who were considered POO or SSC $(n=104), 66 \%$ were referred/known to the community SPC nursing team. The median number of consultations per patient was 2.9, and patients were known to the service for up to five years and two months.

Conclusion COPCCs appears to facilitate collaborative working between disciplines to enable optimal symptom control, information sharing and forward planning to patients attending oncology clinics. Unsurprisingly, PCC focus on symptom control and early referral to SPC services in the community. Many patients are seen when they are 'stable' suggesting the service is proactive in approach. COPCCs remove the need for a 'referral' to SPC, and allows patients to be seen who perhaps wouldn't otherwise be referred to SPC.

\section{P-209 COMBINED ONCOLOGY AND PALLIATIVE CARE CLINICS; HOSPICE IN-PATIENT STAYS AND PLACE OF DEATH (PART 2)}

1,2 Jane Gibbins, ${ }^{2}$ Beccy Benham, ${ }^{2}$ Anna Broadbent, ${ }^{2}$ Amman Mader, ${ }^{1,2}$ Carolyn Campbell, ${ }^{3}$ Rachel Newman, ${ }^{1,2}$ Deborah Stevens, ${ }^{3}$ Joanne Palmer. ${ }^{1}$ Cornwall Hospice Care, St Austell, UK; ${ }^{2}$ Exeter Medical School, Exeter, UK; ${ }^{3}$ Royal Cornwall Hospital Trust, Truro, UK

\subsection{6/bmjspcare-2017-hospice. 234}

Background Early Specialist Palliative Care (SPC) introduced to patients with advanced lung cancer has recently been proven to enhance quality-of-life and improve survival. Combined Oncology and Palliative Care Clinics (COPCC) have been carried at Royal Cornwall Hospital Trust for over two decades, to enable patients to be reviewed by palliative care consultants whilst attending the Oncology Centre; facilitating the two specialities to run in parallel to improve patient care. The aims of this project were to explore the use of inpatient hospice facilities of all patients, and place of death those for those patients who died.

Methods A retrospective case note review of 150 consecutive patients reviewed between 01/2016-06/2016. Data collected by three medical students.

Results Admissions to hospice: 18/150 patients (12\%) had at least one hospice admission, with the most common primary reason for admission being symptom control (83\%). Total number of admissions of any one patient ranged from one to seven. Length of stay from one to 46 days, with a median value of nine days. 11/18 admitted patients died during their most-recent hospice admission, and seven were discharged home. Place of death: 29/150 (19\%) patients died at the time of data collection; $12(41 \%)$ died at home, $11(38 \%)$ died in a hospice, $6(21 \%)$ died in acute $(3 / 10.5 \%)$ or community hospital $(3 / 10.5 \%)$.

Conclusion Access to SPC in COPCCs appears to enable early contact with inpatient hospice units, and enable more patients to die in the hospice and at home. 38\% of this study's deceased patients died in a hospice; a 2.3 fold increase compared to hospice deaths of cancer patients specifically (16.4\%). The number of deaths is small and warrants further studies. 


\section{P-210 MAN SHED - EXTENDING THE REACH AND ENGAGING} THE COMMUNITY

Kathy Birch, Martin Osborn, Julia Russell. Princess Alice Hospice, Esher, UK

\subsection{6/bmjspcare-2017-hospice.235}

Background Historically certain groups of people have been less likely to access hospice support, for example of every three women accessing bereavement support only one man did. Men's Sheds have grown in popularity but only one hospice had a shed specifically for people affected by life-limiting illnesses.

Aims

- To provide alternative support for patients, carers and bereaved relatives

- To diversify volunteering roles

- To demystify hospice care by engaging the local community.

Methods A cross-organisational steering group of staff, volunteers and users was formed and a fundraising project launched which exceeded $£ 112000$. Plans were agreed and a $12.5 \mathrm{~m} \times$ $5.5 \mathrm{~m}$ structure was built by contractors to accommodate a fully functioning wood workshop and social area designed and fitted out by volunteers. Health and Safety, Facilities, Volunteering and Education teams supported induction processes, development of risk assessments and training.

Results

- Cross-organisational project brought together clinical and non-clinical staff and volunteers

- New roles have been created for volunteers

- New links have been forged with businesses and the local community donating wood and consumables

During the first nine months:

- six volunteers have been recruited and trained to lead sessions

- one volunteer provides administrative support, one volunteer ambassador sources equipment and consumables

- Opens three days a week

- 53 'shedders' have been referred, resulting in 357 attendances.

- Patients, carers and bereaved relatives have attended woodworking sessions

- A basic computer literacy course has taken place

- Feedback is positive and the service valued by people who would not have accessed traditional services

- Items produced have raised funds to sustain the project

- A local woodcarving group also uses the building.

Conclusions The Man Shed has created opportunities for people to be offered 'shoulder-to-shoulder' support while engaging in practical activities. This informal approach has helped to break down pre-conceived barriers and misconceptions about hospice care. A number of other hospices are now planning their own sheds.

\section{P-211 THE NURSE LED MODEL OF HOSPICE INPATIENT CARE - 7 YEARS ON}

Dinah Hickish. St Kentigern Hospice, St Asaph, UK

10.1136/bmjspcare-2017-hospice.236
Hospices across the UK are having to re-examine the way their services are run as pressure on funding is becoming increasingly problematic in a nation that has fiscal uncertainty. In Wales statutory funding for hospices is arrived at by a formula and St Kentigern Hospice receives 18\% of its funding from the Welsh Office.

The hospice hit financial crisis in 2010 and took drastic measures with swingeing cuts to services, including the decision to make the medical director and sole doctor redundant. A decision was made that St Kentigern Hospice would become a nurse- led unit in the faint hope that this would save the hospice. This was entirely a reactive decision and could only be made because the hospice had a prescribing Advanced Clinical Practitioner, who had been practising for 18 months in that role.

One year later in 2011, the hospice was fully operational in both its inpatient and day therapies services and $£ 1,000,000$ credit in the bank. This is the story of innovation in practice where the advanced nurse practitioner role in palliative care has been extended to an autonomous level not practised anywhere else in the UK. From adversity this eightbedded IPU and day therapies hospice now has a service that is economically robust and has been approved and recognised by the Welsh peer review process. Such is the success of the model that the hospice is undergoing a major new build, and expansion of services, funded entirely from its own resources, responding further to our communities' palliative care needs.

The model and its sustainability along with challenges will be discussed. Change in practice, innovation and leadership for nursing within a hospice setting will be explored.

\section{P-212 IMPROVING THE PATIENT'S JOURNEY BY DESIGNING AND IMPLEMENTING A BESPOKE, DEDICATED TRIAGE SERVICE}

Elaine Green, Rebecca Chambers. Ashgate Hospicecare Chesterfield, UK

\subsection{6/bmispcare-2017-hospice.237}

Background A comprehensive review was carried out in 2013/ 2014 with the support of Macmillan Cancer Support focusing on workforce development for the Palliative Care Specialist Nurse team working in the community. It recommended the provision of a more responsive and accessible service for patients, carers and health are professionals by developing a bespoke, dedicated Triage and 24/7 Advice Line.

Aims To analyse and explore the most effective referral process to deliver a more responsive and accessible service for all patients who are referred to the Palliative Care Specialist Nurse team.

Method In September 2016 a two-year project commenced which involved four phases:

- Understanding current practices

- Analysis of the literature, audits and clinical practice in other hospices

- The transformational phase which included the development of a bespoke robust model, implementation of systems and processes ready for Test and Launch

- Evaluation of the model, impact analysis on the organisation, patient and carer outcomes.

Results The results of the test in May 2017 demonstrated a clear need for a dedicated Triage referral service Monday to 